\title{
Clones in space-how sampling can bias genetic diversity estimates in corals: editorial comment on the feature article by Gorospe et al.
}

\author{
Cynthia Riginos
}

Published online: 10 March 2015

(C) Springer-Verlag Berlin Heidelberg 2015

Corals, like most colonial animals, are prone to asexual reproduction via fragmentation that creates clonal copies. Genetic studies have consistently shown that asexual reproduction is very common; consequently, coral colonies from the same reef (or nearby reefs) often have identical genotypes. A growing number of studies have formally quantified the genetic similarity among proximate colonies (i.e., positive spatial autocorrelation, for example: Miller 1998; Costantini et al. 2007; Underwood et al. 2007; Miller and Ayre 2008; Underwood et al. 2009; Gorospe and Karl 2013; Smilansky and Lasker 2014). Given that clonality appears to be ubiquitous for corals and that closely related colonies (including clonal copies) tend to be nearby, how does spatial genetic structure (especially small-scale positive spatial autocorrelation) affect common metrics of genetic diversity? And, how difficult is positive spatial autocorrelation to detect?

In the present paper, Gorospe et al. (2015) demonstrate that spatial genetic structure within reefs should be a concern to coral geneticists seeking to describe genetic diversity either within reefs or between reefs (that is, to estimate population genetic structure or gene flow). Moreover, for a number of realistic scenarios, they show that the power to detect small-scale positive spatial autocorrelation is quite low; thus, failure to detect a spatial autocorrelation pattern will not constitute strong evidence for the absence of such a pattern. These issues are tangibly illustrated using their own data (Gorospe and Karl 2013) where they had exhaustively

Communicated by U. Sommer.

C. Riginos $(\bowtie)$

School of Biological Sciences, The University of Queensland, St. Lucia, QLD 4072, Australia

e-mail: c.riginos@uq.edu.au genotyped all 2352 colonies of the "cauliflower" coral, Pocillopora damicornis (using 6 microsatellite loci), from a small patch reef in Kāne`ohe Bay, Hawai'i (Fig. 1). Simulations that vary the degree and proportions of clones within hypothetical populations augment these empirical observations to highlight the generality of the findings.

The results are sobering for any empirical geneticist reflecting upon either past work in corals or embarking upon future studies. First, sampling that is truly random is the most reliable: the more expedient strategies of saturation and nearest neighbor sampling yield diversity estimates with large variance and in some instances bias. Thus, semi-haphazard sampling that typifies many studies-collecting fragments from colonies here and there-is likely to yield biased or unreliable estimates of genetic diversity. Second, huge numbers of individuals need to be genotyped to produce accurate estimates, with lower bounds of 500 to estimate the true proportions of unique genotypes $(\mathrm{G} / \mathrm{N})$ and lower bounds of 1000 or more to detect within-reef spatial autocorrelation patterns.

Why do these results matter? Because corals are the foundational species of coral reefs and sensitive to changes in temperature, sediments, $\mathrm{pH}$, etc., many studies seek to identify populations containing genetic variation that could buffer corals against such stressors or might even be candidate sources for assisted migration. Within-population diversity might also be used to infer populations that have been historically stable and environmental factors that promote stability and persistence. Such inferences based on empirical observations of genetic diversity will be unreliable if sampling lacks the power to estimate true population values. Furthermore, most metrics that describe population genetic structure rely on within-population diversity estimates. If diversity within populations is overestimated, the between-population diversity will be underestimated 
Fig. 1 Reef 19 in Kane'ohe Bay, Hawai'i where Gorospe and Karl conducted the research. Photograph by Kelvin Gorospe

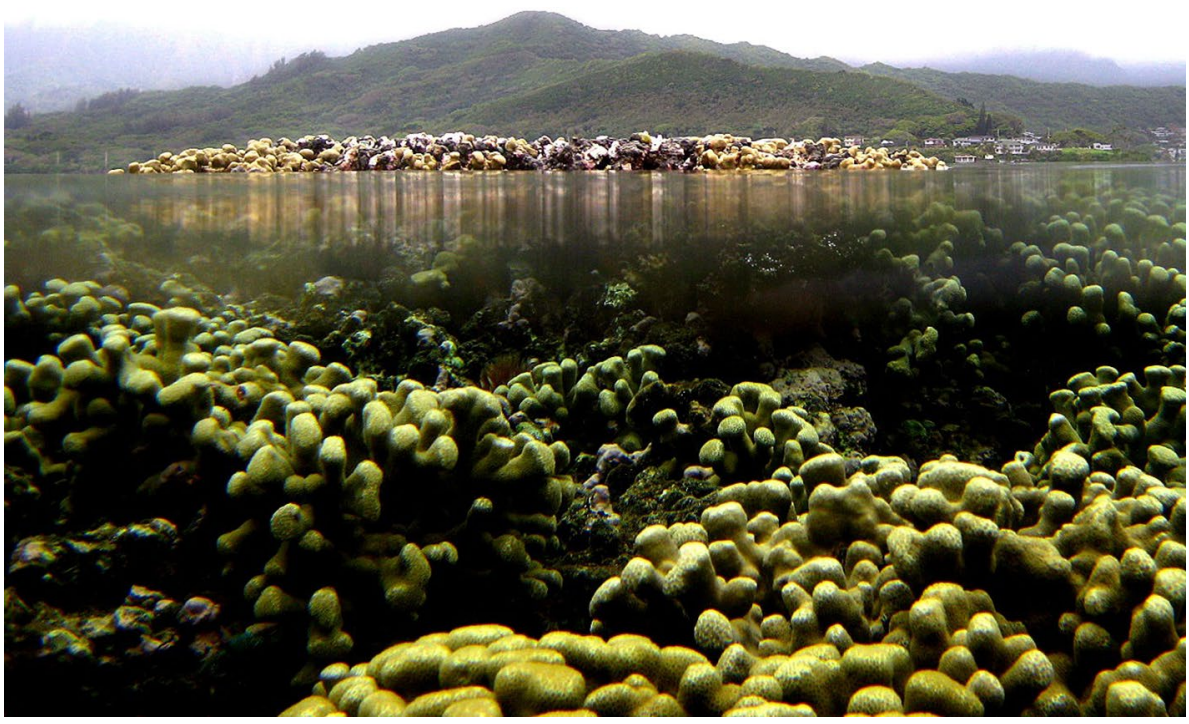

(and gene flow overestimated) and vice versa (Edwards and Beerli 2000). Thus, typical population genetic measures could be especially error prone (and perhaps biased) for corals. Therefore, statistical analyses and interpretation of coral population genetic diversity should, ideally, consider how spatial genetic structure might affect outcomes (and concomitantly implement appropriate field sampling methods including random sampling and large sample sizes).

Although the emphasis of Gorospe et al. (2015) is on corals, intriguingly the authors suggest that positive spatial autocorrelation at short distances might be common in marine organisms, albeit subtle and difficult to detect. Positive spatial autocorrelation at very small distances $(<100 \mathrm{~m})$ has been reported for a number of marine taxa, notably those low dispersal ability (e.g., seagrasses: Alberto et al. 2005; Migliaccio et al. 2005; Ruggiero et al. 2005, sponges: Calderon et al. 2007; Blanquer et al. 2009, bryozoans: Pemberton et al. 2007, and ascidians: Yund and O'Neil 2000; David et al. 2010), but whether positive spatial autocorrelation at relatively short distances is also common for taxa with extended larval durations is unknown. As demonstrated by all the aforementioned studies, incorporating spatially explicit approaches adds a valuable dimension to marine genetic research and can reveal subtle but important patterns of genetic diversity.

\section{References}

Alberto F, Gouveia L, Arnaud-Haond S, Perez-Llorens JL, Duarte CM, Serrao EA (2005) Within-population spatial genetic structure, neighbourhood size and clonal subrange in the seagrass Cymodocea nodosa. Mol Ecol 14:2669-2681

Blanquer A, Uriz MJ, Caujape-Castells J (2009) Small-scale spatial genetic structure in Scopalina lophyropoda, an encrusting sponge with philopatric larval dispersal and frequent fission and fusion events. Mar Ecol Prog Ser 380:95-102

Calderon I, Ortega N, Duran S, Becerro M, Pascual M, Turon X (2007) Finding the relevant scale: clonality and genetic structure in a marine invertebrate (Crambe crambe, Porifera). Mol Ecol 16:1799-1810

Costantini F, Fauvelot C, Abbiati M (2007) Fine-scale genetic structuring in Corallium rubrum: evidence of inbreeding and limited effective larval dispersal. Mar Ecol Prog Ser 340:109-119

David GK, Marshall DJ, Riginos C (2010) Latitudinal variability in spatial genetic structure in the invasive ascidian, Styela plicata. Mar Biol 157:1955-1965

Edwards SV, Beerli P (2000) Gene divergence, population divergence, and the variance in coalescence time in phylogeographic studies. Evolution 54:1839-1854

Gorospe KD, Karl SA (2013) Genetic relatedness does not retain spatial pattern across multiple spatial scales: dispersal and colonization in the coral, Pocillopora damicornis. Mol Ecol 22:3721-3736

Gorospe KD, Donahue MJ, Karl SA (2015) The importance of sampling design: spatial patterns and clonality in estimating the genetic diversity of coral reefs. Mar Biol. doi:10.1007/s00227-015-2634-8

Migliaccio M, De Martino F, Silvestre F, Procaccini G (2005) Meadow-scale genetic structure in Posidonia oceanica. Mar Ecol Prog Ser 304:55-65

Miller KJ (1998) Short-distance dispersal of black coral larvae: inference from spatial analysis of colony genotypes. Mar Ecol Prog Ser 163:225-233

Miller KJ, Ayre DJ (2008) Population structure is not a simple function of reproductive mode and larval type: insights from tropical corals. J Anim Ecol 77:713-724

Pemberton AJ, Hansson LJ, Craig SF, Hughes RN, Bishop JDD (2007) Microscale genetic differentiation in a sessile invertebrate with cloned larvae: investigating the role of polyembryony. Mar Biol 153:71-82

Ruggiero MV, Reusch TBH, Procaccini G (2005) Local genetic structure in a clonal dioecious angiosperm. Mol Ecol 14:957-967

Smilansky V, Lasker HR (2014) Fine-scale genetic structure in the surface brooding Caribbean octocoral, Antillogorgia elisabethae. Mar Biol 161:853-861

Underwood JN, Smith LD, Van Oppen MJH, Gilmour JP (2007) Multiple scales of genetic connectivity in a brooding coral on isolated reefs following catastrophic bleaching. Mol Ecol 16:771-784 
Underwood JN, Smith LD, van Oppen MJH, Gilmour JP (2009) Ecologically relevant dispersal of corals on isolated reefs: implications for managing resilience. Ecol Appl 19:18-29
Yund PO, O’Neil PG (2000) Microgeographic genetic differentiation in a colonial ascidian (Botryllus schlosseri) population. Mar Biol 137:583-588 